\title{
Object-load and feature-load modulate EEG in a short-term memory task
}

\author{
Niko A. Busch ${ }^{1,2, C A}$ and Christoph S. Herrmann ${ }^{1,2}$ \\ 'University of Magdeburg, Department of Biological Psychology, Postfach 4120, 39016 Magdeburg, Germany; ${ }^{2}$ Max Planck Institute of Cognitive \\ Neuroscience, Postfach 500355, 04303 Leipzig, Germany \\ ${ }^{\mathrm{CA} C}$ orresponding Author: niko.busch@nat.uni-magdeburg.de
}

Received 6 May 2003; accepted 8 June 2003

DOI: 10.1097/0I.wnr.0000087727.58565.lb

\begin{abstract}
Behavioral studies have indicated that multiple features of one object can be stored in working memory without additional costs. In contrast, visual search experiments revealed that search for a multi-featured object takes more time than for a single-featured object. We used EEG to differentiate the effect of object-load and feature-load in a short-term memory task. We independently varied the amount of objects and features that had to be memorized. Object-load modulated P3 amplitude during encoding and induced
\end{abstract}

$10 \mathrm{~Hz}$ oscillations during the retention interval. Feature-load modulated the $\mathrm{P} 3$ during retrieval. Thus, only object-load seemed to influence encoding and retention while feature-load played a crucial role during retrieval. Our results demonstrate that objectload and feature-load influence short-term memory at different stages. NeuroReport 14:1721-1724 (c) 2003 Lippincott Williams \& Wilkins.

Key words: EEG; ERP; Feature integration; $10 \mathrm{~Hz}$; P3; Memory load; Short-term memory

\section{INTRODUCTION}

According to feature integration theory (FIT), visual information is processed in at least two successive stages [1]. In a first stage the presence of elementary stimulus attributes (or features) such as color, spatial orientation or shape is detected and coded in so called feature maps. The theory hypothesizes separate maps for different stimulus dimensions. This idea receives support from neurophysiological studies which show that visual information is processed in a network of different brain areas specialized in the processing of separate stimulus attributes [2]. If a stimulus is defined by more than one feature the problem of integrating the separate features arises [3]. This is accomplished in a second step in which an attentional resource is needed to bind coherent features together. This theory has been tested in visual search paradigms $[1,4,5]$. In a typical experiment subjects have to search a display for a target stimulus that is hidden among distractors. If the target is defined by only one feature (e.g. a red stimulus among green distractors) search times usually do not increase with the number of distractors. If the target is defined by a conjunction of two features (e.g. a red $X$ among green $X s$ and red Os) search times increase linearly with the number of distractors [1]. Taken together, visual search experiments suggest that processing multi-featured stimuli is more effortful than processing single-featured stimuli.

A different pattern of results emerges when a stimulus display does not have to be searched but memorized in visual short term memory (VSTM). The capacity of VSTM is known to be limited to only a few items [6]. Recently, Luck and Vogel used multi-featured stimuli in a series of behavioral experiments and demonstrated that capacity is limited only by the number of objects held in VSTM (objectload). Capacity was not limited by the number of object features (feature-load) that had to be memorized [7] (more details given in [8]). For instance, performance did not decrease when subjects had to memorize an object that was defined by four features (orientation, size, color and the presence or absence of a black gap) compared to memorizing an object defined by only a single feature [8] (Experiment 14). Therefore, the authors concluded that VSTM stores integrated objects rather than a set of individual features (e.g. a red square rather than red and square). Thus, unlike visual perception, the retention of objects in VSTM does not seem to be more effortful for multi-featured stimuli than for single-featured stimuli.

One severe limitation of behavioral measures is that they usually represent the combined output of multiple subprocesses. Thus, it cannot be excluded from the behavioral studies done so far that some subprocesses of VSTM work quite similar to visual perception while others may not. Therefore, electrophysiological measures might be better suited to resolve the apparent divergence between findings from VSTM and visual perception because of their better temporal resolution.

Previous studies investigated the effect of memory load on event-related potentials (ERPs) and event-related oscillations (EROs). A common finding is a reduction of $\mathrm{P} 3$ 
amplitude with increasing memory load [9-11]. An interpretation given by Kok [12] is that increasing the cognitive demands by increasing memory load leaves fewer resources for stimulus evaluation which is reflected by the P3. EROs in the alpha band $(\sim 10 \mathrm{~Hz})$ were found to increase with memory load during the delay interval in a working memory task $[9,13]$. This increase in $10 \mathrm{~Hz}$ activity has been interpreted as a correlate of a cortical inhibition of taskirrelevant neural processes [14-16]. While most of these studies employed only single-featured objects, Klaver et al. [11] used multi-featured objects to investigate the effect of feature-load on ERPs during encoding and retention and failed to find an effect of feature-load.

The present study aimed at extending previous results by investigating the effect of both object-load and feature-load. For the first time we investigated the effect of both load factors on ERPs as well as on EROs during all stages of a delayed-matching-to-sample task (encoding, retention and retrieval). Since behavioral measures were not influenced by feature-load in the studies conducted by Luck and Vogel [8] one could expect electrophysiological measures to be also modulated by object-load only.

\section{MATERIALS AND METHODS}

Sixteen paid volunteers (mean age 24.6 years, eight female) participated in the study. All subjects gave written informed consent. The experiment consisted of four blocks with 100 trials each. Prior to the recording a training block was given with 20 sample trials for each task. A trial always started with a display of three objects (S1; size $4.5^{\circ}$ visual angle; duration $1000 \mathrm{~ms}$; see Fig. 1) that were presented centrally. During the delay interval a blank screen was shown for $3000 \mathrm{~ms}$ followed by a probe display containing a single object (S2; size $2^{\circ}$ visual angle; duration $1000 \mathrm{~ms}$ ). Subjects were instructed to indicate with a button press whether S2 was included in S1 or not. Stimuli were defined by shape (circle, square, triangle, diamond), color (red, green, blue, yellow) and texture (lines rotated by $0^{\circ}, 45^{\circ}, 90^{\circ}$ or $135^{\circ}$ ).

The order of the four blocks was randomized across subjects. Response hand was counterbalanced across subjects. The probability of a new item was always 50\%. Our goal was to keep the displays as comparable as possible in spite of different task instructions. In each of the four blocks the S1 display contained three stimuli. In those blocks in which only one object had to be remembered (1-object conditions) these three stimuli were identical. Additionally, stimuli always consisted of three features each. In those blocks in which only one feature had to be memorized (1-feature conditions) subjects were instructed to only remember the objects' shape. In this case the S2 stimulus could only change in shape but the other irrelevant features always remained the same.

Accordingly, in the 1-object/1-feature condition the S1 consisted of three identical stimuli. Subjects were instructed to only remember the shape of one of the identical objects. The S2 could differ from S1 only in shape. In the 1-object/3features condition the S1 also consisted of three identical stimuli. But here subjects were told to memorize all three features of one of the identical objects. In this condition the S2 could differ from S1 in either shape, color or texture. In the 3-objects/1-feature condition the S1 consisted of three

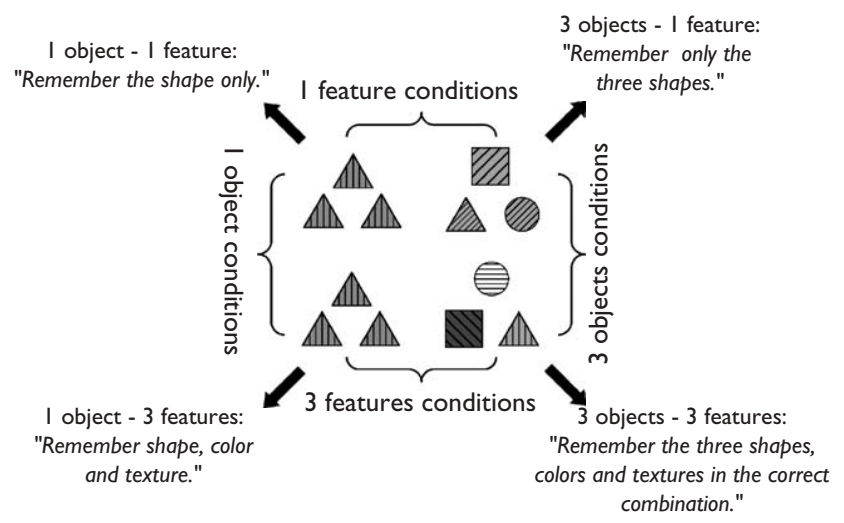

Fig. I. Example of SI stimulus displays used in the four conditions together with the corresponding instructions. Colors are displayed as different shades of grey.

stimuli of different shape but identical color and texture. Subjects had to memorize the three different shapes. The S2 could show a new shape but the other irrelevant features never differed from S1. Finally, in the 3-objects/3-features condition the S1 consisted of three stimuli differing in shape, color and texture. Subjects had to memorize all features of all three stimuli. Additionally, subjects were instructed to also retain the correct combinations of features. The S2 stimulus could differ from S1 in that it either contained one new feature that was not contained in S1 or in presenting a new combination of features which originally belonged to different objects.

EEG was recorded with TMS amplifiers (Twente Medical Systems, Enschede, The Netherlands) using $19 \mathrm{Ag}-\mathrm{AgCl}$ electrodes mounted in an elastic cap at the standard 10/20 positions. All electrodes were referenced to the left mastoid. Electrode impedances were kept $<5 \mathrm{k} \Omega$. Horizontal and vertical EOG were registered with four additional electrodes. Data were sampled at $500 \mathrm{~Hz}$ and analog-filtered with a $0.1 \mathrm{~Hz}$ high-pass and a $100 \mathrm{~Hz}$ low-pass filter. Additionally, ERPs were low-pass filtered digitally at $20 \mathrm{~Hz}$ for display. Electrodes were pooled to five topographical regions of interest (ROIs). The left anterior region (LAR) was comprised of electrodes FP1, F3, F7 and T7. The left posterior region (LPR) included electrodes $\mathrm{P} 3, \mathrm{P} 7$ and $\mathrm{O} 1$. The respective regions over the right hemisphere included the homologous electrodes. The central region (CER) was comprised of electrodes FZ, CZ, PZ, C3 and C4.

We computed induced $10 \mathrm{~Hz}$ activity as the sum of phaselocked and non-phase-locked oscillatory activity with a wavelet-based time-frequency analysis [17,18]. Averages were computed for the S1 interval $(200 \mathrm{~ms}$ before to $800 \mathrm{~ms}$ after S1 onset), the delay interval ( $200 \mathrm{~ms}$ before to $3000 \mathrm{~ms}$ after S1 offset), and the S2 interval ( $200 \mathrm{~ms}$ before until $800 \mathrm{~ms}$ after S2 onset). For artefact rejection, trials were automatically excluded from averaging, if the standard deviation within a moving $200 \mathrm{~ms}$ time interval exceeded $30 \mu \mathrm{V}$ in any electrode. After automatic rejection trials were visually inspected and rejected if eye movements or electrode drifts were visible. Trials in which the subject made an incorrect response were also excluded. Two subjects had to be excluded completely from further 


\section{SI: ERPs}

PZ

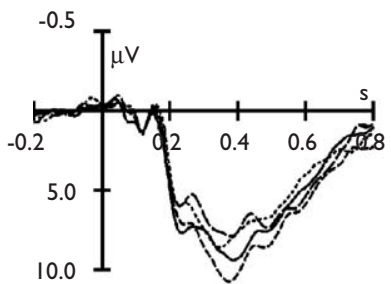

Delay: $10 \mathrm{~Hz}$ oscillations

PZ

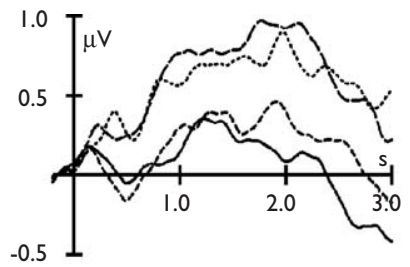

S2: ERPs

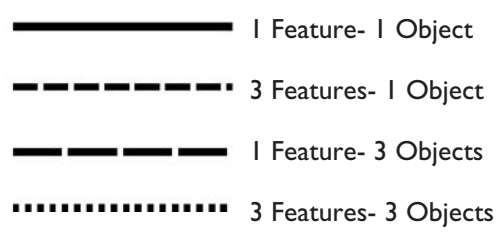

Fig. 2. ERPs during encoding (SI, left) are modulated by object-load. $\mathrm{P} 3$ amplitude is reduced in the 3 -objects conditions. Induced $10 \mathrm{~Hz}$ oscillations during retention (delay, middle) are also modulated by object-load only. The 3 -objects conditions induce larger $10 \mathrm{~Hz}$ amplitudes. At the time of retrieval (S2, right) both factors interact. P3 amplitude is smallest for the most difficult condition (3-features/3-objects).

analysis due to a very poor performance $(<50 \%$ valid trials in one condition).

Repeated measures ANOVAs with the factors featureload and object-load were computed for error rates and reaction times. ERP components were defined as mean amplitudes in the time intervals $200-500 \mathrm{~ms}$ after S1 stimulus onset (encoding-P3) and $400-700 \mathrm{~ms}$ after S2 stimulus onset (retrieval-P3). Induced $10 \mathrm{~Hz}$ oscillations in the delay interval were analyzed in a time window from S1 offset until S2 onset $(3000 \mathrm{~ms})$. The ANOVA for the ERP components and the $10 \mathrm{~Hz}$ oscillations comprised the factors feature-load and object-load. The ANOVA for $10 \mathrm{~Hz}$ oscillations also comprised the factor ROI while for P3 amplitudes only CER was analyzed. All effects with more than two degrees of freedom in the enumerator were adjusted for violations of sphericity and Greenhouse-Geisser epsilons were used to compute $p$.

\section{RESULTS}

The ANOVA for the error rates yielded significant main effects for feature-load $(\mathrm{F}(1,13)=143.41, p<0.001)$ and object-load $(\mathrm{F}(1,13)=77.78, p<0.001)$ as well as an interaction between feature- and object-load $(F(1,13)=54.85$, $p<0.001)$. These effects were largely due to an increase in errors in the 3-features/3-objects condition. Post-hoc tests revealed that feature-load only yielded a significant effect for the 3-object conditions $(\mathrm{F}(1,13)=79.3, p<0.001 ; 1$ feature/1-object: $6.3 \%$; 3-features/1-object: $8.5 \%$; 1-feature/ 3-objects: $9.3 \%$; 3-features/3-objects: $34.1 \%$ ).

An ANOVA for reaction times also yielded significant main effects for feature-load $(\mathrm{F}(1,13)=16.71, p=0.001)$ and object-load $(\mathrm{F}(1,13)=12.56, p=0.004)$. The feature-load $\times$ object-load interaction was also significant $(F(1,13)=10.01$, $p=0.007)$. Post-hoc tests revealed that these results were largely influenced by the longer RT in the 3-features/ 3-objects condition. The post-hoc ANOVA only yielded a significant effect for feature-load in the 3-objects conditions $(\mathrm{F}(1,13)=16.83, p=0.001 ; 1$-feature/1-object: $512 \mathrm{~ms} ; 3$-features/1-object: $540 \mathrm{~ms}$; 1-feature/3-objects: $547 \mathrm{~ms}$; 3-features/3-objects: $666 \mathrm{~ms}$ ).

Figure 2 shows the ERPs in response to S1 and S2 stimuli and induced $10 \mathrm{~Hz}$ oscillations during the delay interval. For the amplitude of the encoding-P3 the ANOVA yielded a main effect for object-load only $(\mathrm{F}(1,13)=6.61, p=0.023)$ indicating smaller amplitudes in the 3-objects conditions.

Amplitude of induced $10 \mathrm{~Hz}$ activity during the delay interval yielded significant effects of ROI $(\mathrm{F}(4,52)=4.39$, $p=0.038)$ and object-load $(\mathrm{F}(1,13)=5.43, p=0.036)$. In addition there was a significant ROI $\times$ object-load interaction $(\mathrm{F}(4,52)=4.25, p=0.021)$. This interaction was further examined in separate post-hoc tests for each ROI. No load effect was observed in ALR and in ARR. In PLR there was a main effect for object-load with higher activity for the 3objects conditions $(\mathrm{F}(1,13)=6.81, p=0.022)$ whereas no load-effect occurred in PRR. In CER the post-hoc test also revealed a main effect for object-load $(F(1,13)=7.20$, $p=0.019)$, again with higher activity in the 3-objects conditions.

The ANOVA of retrieval-P3 amplitudes yielded a main effect for object-load $(\mathrm{F}(1,13)=22.41, \quad p<0.001)$ indicating smaller amplitudes in the 3-objects conditions, and an object-load $\times$ feature-load interaction $(\mathrm{F}(1,13)=27.00$, $p<0.001)$. Post-hoc tests revealed a significant feature-load effect in the 3 -objects conditions only $(\mathrm{F}(1,13)=15.96$, $p=002)$.

\section{DISCUSSION}

Our study revealed a significant modulation of encoding-P3 and $10 \mathrm{~Hz}$ oscillations by object-load during the retention interval but failed to show an effect of feature-load. This is in line with previous results from behavioral [8] as well as electrophysiological studies [11-13]. 
The smaller amplitude of encoding-P3 in the 3-objects conditions irrespective of feature-load indicates that processing multiple features of a stimulus does not require more resources when that stimulus is transferred into VSTM compared to when only a single feature is encoded [12]. This supports the view that VSTM stores integrated object representations rather than several individual features.

The stronger increase in $10 \mathrm{~Hz}$ oscillations for high objectload during the retention interval replicates results from previous studies [9,13]. For instance, Jensen et al. [13] found a positive correlation between memory load and alpha band power. In a different paradigm Cooper et al. [14] found an increase in alpha oscillations in a mental imagery task compared to an externally directed attention task. This increase has been interpreted as a correlate of inhibition of task irrelevant cortical areas. Thus, our results seem to add to the growing evidence that the alpha rhythm represents more than just a state of cortical inactivity (cortical idling). An oscillatory process as the basis of VSTM maintenance was also suggested by Vogel et al. [8]. If multi-featured objects were stored as separate features in VSTM an increase in feature-load should increase the demand for cortical inhibition of task irrelevant cortical areas. Therefore, one would expect a modulation of $10 \mathrm{~Hz}$ activity by both feature-load and object-load. However, a modulation was found for object-load only, supporting the idea of integrated object representations in VSTM. Several authors discussed an alternative account for the lacking effect of feature-load $[7,19,20]$. According to the multiple resources hypothesis, VSTM consists of multiple subcomponents specialized in the storage of different feature dimensions. A multi-featured stimulus then does not put any more load on VSTM than a single-featured stimulus because every subcomponent has to retain only a small amount of information. But as the amount of activated subcomponents increases with featureload one would also expect an increase in $10 \mathrm{~Hz}$ activity. However, our findings of the $10 \mathrm{~Hz}$ activity during the retention period being unmodulated by feature-load argues against this hypothesis.

During retrieval, when subjects had to compare the content of their memory with the S2 stimulus, retrieval-P3 was significantly smaller in the 3-objects/3-features condition compared to the other conditions. This condition differed from the others in so far as the probe stimulus had to be scanned not only for new features but also for a new combination of old features. In this case FIT predicts a larger effort for feature integration in order to avoid miscombinations of features from different objects (so called illusory conjunctions [21]). Why is this larger effort only present during retrieval? One possible interpretation is that integrated object representations (as seem to occur in VSTM) are less well suited for comparison with a probe stimulus. Therefore, memory representations would have to be recoded to a representational format that requires additional feature binding, especially in the 3-objects/3-features condition in which illusory conjunctions can occur. Such a representation could be compared with the probe stimulus for each feature dimension separately (and may be for feature conjunctions) rather than comparing the memory template as a whole. A feature wise comparison process is also known from stimulus classification tasks [17,18]. For instance, in the experiment by Herrmann et al. [17] a target was defined as an illusory figure (feature one) consisting of four inducer discs (feature two). Reaction times were fastest for stimuli which shared no feature with the target (a nonillusory figure consisting of three inducer discs), intermediate for stimuli sharing one feature with the target and slowest for target stimuli which, by definition, shared both features. Hence, the authors concluded that stimulus and memory template were compared feature by feature. Taken together, our results confirm that storage and retention work with integrated objects in VSTM but suggest that retrieving and comparing the stored memory content relies on the single features of objects. This also explains why visual search yields effects of feature-load while memory experiments often fail to do so. In a visual search experiment the items of the search display have to be compared to the target which was previously encoded and which needs to be retrieved from memory. I.e. the process of retrieval and comparison which yields effects of feature-load is one of the main processes involved in this task which is usually measured by reaction times. In a memory experiment, however, three subsequent tasks add up to one reaction time: encoding, retention and retrieval/comparison. Thus, it is plausible that feature-load is not as crucial since it is involved only in the last of the three processes.

\section{REFERENCES}

1. Treisman AM and Gelade G. Cogn Psychol 12, 97-136 (1980).

2. Livingstone M and Hubel D. Science 240, 740-749 (1988).

3. Robertson LC. Nature Rev Neurosci 4, 93-102 (2003).

4. Wolfe JM. Trends Cogn Sci 7, 70-76 (2003)

5. Wolfe JM, Cave KR and Franzel SL. J Exp Psychol Hum Percept Perform 15, 419-433 (1989).

6. Cowan N. Behav Brain Sci 24, 87-114; discussion 114-115 (2001).

7. Luck SJ and Vogel EK. Nature 390, 279-281 (1997).

8. Vogel EK, Woodman GF and Luck SJ. J Exp Psychol Hum Percept Perform 27, 92-114 (2001).

9. Schack B and Klimesch W. Neurosci Lett 331, 107-110 (2002).

10. McEvoy LK, Smith ME and Gevins A. Cerebr Cortex 8, 563-574 (1998).

11. Klaver P, Smid HG and Heinze HJ. Neurosci Lett 268, 65-68 (1999).

12. Kok A. Psychophysiology 38, 557-577 (2001)

13. Jensen O, Gelfand J, Kounios J and Lisman JE. Cerebr Cortex 12, 877-882 (2002).

14. Cooper NR, Croft RJ, Dominey SJ et al. Int J Psychophysiol 47, 65-74 (2003).

15. Klimesch W, Doppelmayr M, Schwaiger J et al. Brain Res Cogn Brain Res 7, 493-501 (1999).

16. Foxe JJ, Simpson GV and Ahlfors SP. Neuroreport 9, 3929-3933 (1998).

17. Herrmann CS, Mecklinger A and Pfeifer E. Clin Neurophysiol 110, 636-642 (1999).

18. Herrmann CS and Mecklinger A. Int I Psychophysiol 38, 265-281 (2000).

19. Wheeler ME and Treisman AM. J Exp Psychol Gen 131, 48-64 (2002).

20. Olson IR and Jiang Y. Percept Psychophys 64, 1055-1067 (2002).

21. Treisman A and Schmidt H. Cogn Psychol 14, 107-141 (1982).

Acknowledgements: We express our thanks to Cornelia Schmidt for help during data acquisition and to Maren Grigutsch for technical support. This study was supported by the German Research Foundation (DFG, grant HE 3353/2-I). 